\title{
Finalidad de los cursos de adiestramiento práctico
}

\author{
Hernández Fernández C. \\ Servicio de Urología. Hospital General Universitario Gregorio Marañón. Madrid.
}

Actas Urol Esp 2006; 30 (5): 461-463

\section{RESUMEN \\ FINALIDAD DE LOS CURSOS DE ADIESTRAMIENTO PRÁCTICO}

La cirugía laparoscópica se inicia en la década de los 90 y sobre todo se desarrolla con fuerza en los albores de este siglo, es, por tanto, una alternativa quirúrgica muy reciente.

Esto ha supuesto que la formación de cada equipo haya recorrido caminos muy dispares, hoy cuando contamos ya con Servicios expertos es fundamental sistematizar la enseñanza y acortar las curvas de aprendizaje, para ello los cursos de adiestramiento práctico que combinan cirugías en pacientes con cirugias en el quirófano experimental son absolutamente imprescindibles.

Palabras clave: Laparoscopia. Cursos de adiestramiento.

\section{ABSTRACT}

\section{PURPOSE OF THE PRACTICAL TRAINING COURSES}

Laparoscopy surgery started in the 90's decade and it is rapidly developing in the first years of this century; it is therefore a very recent surgical alternative. This has made that each teams formation has gone along very different ways, today that we have already expert services it is essential to systematize the training and to shorten the learning curve. For that purpose we have the practical training courses that combine surgery in patients with surgery in experimental operating theatres, which are absolutely indispensable.

Keywords: Laparoscopy. Training courses.

$\mathrm{L}^{2}$ a formación de especialistas en cirugía laparoscópica es un tema aún por resolver y ello es debido a un cúmulo de factores.

Los pioneros de estas técnicas las fueron desarrollando de forma casi intuitiva y sin seguir ninguna planificación previa. De hecho en nuestro propio país algunos grupos empezaron por la linfadenectomía obturatriz en el cáncer de próstata, otros con la colposuspensión, algunos directamente con la cirugía renal e incluso, más recientemente, con la prostatectomía radical.

Si analizamos este hecho de forma comparativa con la cirugía abierta, sería como decir que en algunas escuelas los cirujanos noveles empiezan su entrenamiento con la cistectomía y derivación urinaria, en otras con el tumor con trombo en cava, en varias por la ureterolitotomía y casual- mente las hay que recomiendan empezar por la fimosis. En resumen un verdadero caos.

Si a ello le añadimos que son escasos los centros y en consecuencia el número de especialistas que tienen ya superada su curva de aprendizaje y que por último, en Urologia, la patología frecuente y sencilla que puede operarse por laparoscopia no existe, la problemática para sistematizar la formación en este abordaje está servida.

Es por este motivo por lo que han surgido múltiples cursos para la formación y entrenamiento en laparoscopia y que podríamos agrupar en tres conceptos fundamentales:

- Cursos experimentales de iniciación

- Cursos avanzados clínico-experimentales

- Cursos de consolidación tutorizados

Nos referiremos en este artículo a los: 


\section{CURSOS CLÍNICO-EXPERIMENTALES}

Objetivo de los cursos

Estos cursos están enfocados para atender a la demanda de los Servicios de Urología del país que han iniciado o están en vías de iniciar la cirugía laparoscópica, y que precisan de una formación práctica que les facilite una curva de aprendizaje habitualmente difícil en nuestra especialidad.

En estos equipos ya existen personas que han demostrado interés en adquirir una formación en cirugía laparoscópica y que, en la mayoría de los casos, han asistido a cursos experimentales de iniciación.

Por lo tanto tienen los conocimientos básicos en cuanto a tecnología, aparataje, abordajes, etc.

\section{Estructura de los cursos}

Son cursos para un reducido número de alumnos, en general entre 6 y 8 , donde se van a combinar cirugias en pacientes, realizadas por especialistas con asegurada experiencia en las mismas, con cirugía en animales de experimentación.

Es fundamental que a los alumnos se les ubique físicamente en el quirófano. De tal manera que esta parte del curso no se limite a ver en el monitor la técnica concreta sino que conozcan y perciban, en directo, el trabajo de enfermería, la colocación del paciente, el posicionamiento de la tecnología a emplear, donde y como se colocan cirujano y ayudantes etc. y puedan, en cualquier momento, preguntar dudas, plantear problemas $y$, sobre todo, salir con la idea de que, al igual que en cirugía convencional, la cirugía laparoscópica es un trabajo en equipo donde la planificación es la norma y la improvisación la excepción.

Como complemento fundamental de esta parte del curso el 50\% del tiempo se ha de dedicar a repetir en animales, habitualmente en cerdos, las técnicas que se vieron hacer previamente en los pacientes. De tal forma que los alumnos, en grupos de dos, y tutelados por expertos, sean capaces de reproducir las mismas maniobras quirúrgicas, utilizar similar tecnología, solventar similares complicaciones, etc.

No se debe olvidar que ha de dedicarse también un tiempo a mejorar la destreza laparoscópica con el trabajo en simuladores. El experto en cirugía laparoscópica gracias al entrenamiento compensa, con la experiencia, la pérdida de la visión tridimensional sin que ello suponga una limitación para la precisión de movimientos. Por lo tanto las horas pasadas en el simulador trabajando con pinzas, tijeras, portas, etc, permiten automatizar movimientos claves para suturar, anudar, seccionar, etc.

Otro de los objetivos de este tipo de cursos es el de enseñar y poner en manos de los alumnos los distintos tipos de tecnología que pueden facilitar las técnicas quirúrgicas, y más si cabe, en la curva de aprendizaje. Si nos centramos en la cirugía renal se ha de enseñar a trabajar con energía mono y bipolar, sellador de vasos, productos hemostáticos, etc. Que duda cabe que no es imprescindible tener todos pero sí conocerlos para luego poder optar por aquellos que parezcan más interesantes en la futura práctica clínica. En los cursos que impartimos en el Hospital Gregorio Marañón desde hace cuatro años se trabaja con ligasure, coagulador de argón, energía mono y bipolar, etc.

Pero tal vez lo más importante es el poder trasmitir a los que se inician en este abordaje la sistemática de las distintas técnicas quirúrgicas, ya que, al igual que en cirugía convencional, la secuencia de gestos quirúrgicos ha de estar perfectamente definida. Se ha demostrado que, en el aprendizaje de la laparoscopia, la mayor parte de los errores son consecuencia de tres tipos de equivocaciones: omisión de pasos quirúrgicos fundamentales, pasos quirúrgicos dados con una secuencia errónea o bien utilizar con excesiva fuerza el instrumental. Todo esta dinámica se ha de aprender en este tipo de cursos.

Es por último recomendable, puesto que la duración de los mismos no suele ser más de tres días, que se dediquen de forma monográfica a una región anatómica, bien sea próstata, riñón, etc., ya que la posición de los pacientes, la colocación de los puertos de entrada, la situación de cirujano y ayudante, etc. son distintos y si se intentan enseñar todos, en tan poco tiempo, pueden confundir más que mejorar la curva de aprendizaje.

Se han publicado varios trabajos en los que se intenta sistematizar la enseñanza de la laparoscopia y algunos de ellos comparan diferentes 
modelos de entrenamiento, con animales, con simuladores, etc., incluso apuntando que la formación con modelos computarizados es similar, en resultados, al trabajo hecho en el quirófano experimental, sin embargo la conclusión más unánime en la mayoría de los estudios piloto es que, la formación del urólogo que desea aprender las técnicas laparoscópicas, debe incluir modelos inanimados, entrenamiento con animales y completado con cirugías en humanos dirigidos por especialistas ya expertos.

Con este análisis no queremos concluir que son suficientes estos cursos para superar la curva de aprendizaje, sino que son un peldaño más en un proceso de formación en cirugía laparoscópica siempre lento, a veces tedioso, pero que permitirá alcanzar la destreza necesaria para operar a los pacientes controlando al máximo los riesgos que cualquier técnica conlleva.

\section{BIBLIOGRAFIA RECOMENDADA}

1. Roser JC, Roser LE. Salvagi RS. Skill acquisition and assessment for laparoscopic surgery. Arch Surg. 1997; 132:200-204.

2. Traxer O, Gettman MT, Napper CA, Scott DJ, Jones DB, Roehrborn CG, et al. The impact of intense laparoscopic skills training on the operative performance of urology residents. J Urol. 2001;166(5):1658-1661.
3. Cadeddu JA, Wolfe JS Jr, Nakada S, Chen R, Shalhav A, Bishoff JT, et al. Complications of laparoscopic procedures after concentrated training in urological laparoscopy. J Urol. 2001;166(6):2109-2111.

4. Novas S, Ruibal M, González M. Docencia en urología laparoscópica. Problemas. Soluciones. Actas Urol Esp. 2005;29 (3):241-243.

5. Tang B, Hanna GB, Cuschieri A. Analisis of errors enacted by surgical trainers during skills training courses. Surgery. 2005; 138(1):14-20.

6. Martín RC, Kehdy FJ, Allen JW. Formal training in advanced surgical technologies enhances the surgical residency. Am J Surg 2005; 190(2):244-248.

7. Sharpe BA, MacHaidze Z, Ogan K. Randomized comparison of standard laparoscopic trainer to novel, at-home,low-cost, camera-less laparoscopic trainer. Urology. 2005;66(1):5054.

8. Modam AK, Franzides ST, Tebbit C, Quiros RM. Participants opinions of laparoscopic training after a basic laparoscopic training course. Am J Surg 2005;189(6):758-761.

9. Melvin VS, Jonson JA, Ellison EC. Laparoscopic skills enhancement. Am J Surg 1996;172:377-381.

Dr. C. Hernández Fernández

Servicio de Urología

Hospital General Univ. Gregorio Marañón

Doctor Esquerdo, 46

28007 Madrid

E-mail: chdez@pulso.com 